\title{
THE ACCELERATION OF POLARIZED PROTONS IN CIRCULAR ACCELERATORS
}

E.D. COURANT and R.D. Ruth

September 12, 1980

ACCELERATOR DEPARTMENT

BROOKHAVEN NATIONAL LABORATORY ASSOCIATED UNIVERSITIES, INC. UNDER CONTRACT NO. DE-AC02-76CHO0016 WITH THE UNITED STATES DEPARTMENT OF ENERGY 


\section{DISCLAIMER}

This report was prepared as an account of work sponsored by an agency of the United States Government. Neither the United States Government nor any agency Thereof, nor any of their employees, makes any warranty, express or implied, or assumes any legal liability or responsibility for the accuracy, completeness, or usefulness of any information, apparatus, product, or process disclosed, or represents that its use would not infringe privately owned rights. Reference herein to any specific commercial product, process, or service by trade name, trademark, manufacturer, or otherwise does not necessarily constitute or imply its endorsement, recommendation, or favoring by the United States Government or any agency thereof. The views and opinions of authors expressed herein do not necessarily state or reflect those of the United States Government or any agency thereof. 


\section{DISCLAIMER}

Portions of this document may be illegible in electronic image products. Images are produced from the best available original document. 


\title{
THE ACCELERATION OF POLARIZED PROTONS IN CIRCULAR ACCELERATORS
}

\author{
E.D. COURANT AND R.D. RUth
}

September 12, 1980

\section{ACCELERATOR DEPARTMENT}

BROOKHAVEN NATIONAL LABORATORY UPTON, NEW YORK 11973 


\section{DISCLAIMER}

This Ir.] States Government. Neither the United States Government nor any agency thereof,

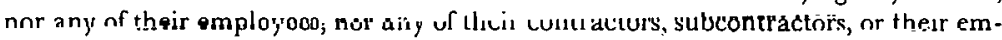
ployees, makes any warranty, express or implied, or assumes any legal liability or responsibility for the accuracy, completeness, or usefulness of any information, apparauus, pruduct, or prócèss disclosed, or represents that its use would not infringe pri-

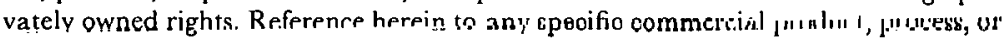
service by trade name, trademark, manufacturer, or otherwise, does not necessarily constitute or imply its endorsement, recommendation, or favoring by the United States Government or any agency, contractor or subcontractor thereof. The views and opinions of authors expressed herein do not necessarily state or reflect those of the United States Government or any agency, contractor or subcontractor thereof.

Printed in the United States of America

Available from

National Technical Information Service

U.S. Department of Commerce

5285 Port Royal Road

Springfield, VA 22161

Price: Printed Copy $\$ 6.00$; Microfiche $\$ 3.50$ 


\section{ABSTRACT}

In this paper we present the theory of depolarization in circular accelerators. The spin equation is first expressed in terms of the particle orbit and then converted to the equivalent spinor equation. We then solve the spinor equation for three different situations: 1) A beam on a flat top near a resonance, 2) Uniform acceleration through an isolated resonance, and 3) A model of a fast resonance jump. Finally, we calculate the depolarization coefficient, $\varepsilon$, in terms of properties of the particle orbit and apply the results to a calculation of depolarization in the AGS. 


\section{INTRODUCTION}

The problem of acceleration of polarized particles has been analyzed by numerous authors,,$^{1-5}$ and polarized protons have, in fact, been successfuliy accelerated to $12.75 \mathrm{GeV}$ at the Argonne ZGS. ${ }^{6}$ The major problem is the resonant depolarization that occurs when the perturbing fields, as seen by the particles, contain components with frequency equal to the spin precession frequency. These resonances occur whenever the spin precession frequency, $x=\gamma G$, is a combination of the frequency of oscillation of the particle and the periodic spatial variation of the guide field, i.e. when

$$
x=k \text { or } k \pm v
$$

where $\mathrm{k}$ is an integer and $\nu$ is the Betatron oscillation "tune" (the betatron oscillation frequency in units of the revolution frequency). $\quad \gamma$ is the relativistic energy factor, and $G$ is the anomalous magnetic moment coefficient

$$
G=g / 2-1
$$

Higher order resonances, where $v$ in the above equation is replaced by a linear combination of the frequencies of the three degrees of freedom, may sometimes be important but are not considered here.

The aim of the present paper is to present in detail the theory of depolarization resonances in a fashion applicable to any proton accelerator, to express the results in terms of the properties of the particle arhit. (similar to Courant ${ }^{3}$ and Ernst ${ }^{4}$ ), and to obtain expressions suitable for numerical calculations. Part of this paper is an elaboration of a short paper which appeared previously, ${ }^{7}$ and some of the treatment here is equivalent to that given elsewhere. 
II THE SPIN EQUATIONS

Froissart and Stora ${ }^{1}$ have shown with the theory of Bargmann, Michel and Telegdi, 8 that the spin of a particle taken as a classical normalized vector $\vec{S}$ in a static magnetic field obeys the equation:

$$
\frac{d \vec{S}}{d t}=\frac{e}{\gamma c} \vec{S} \times\left[(1+\gamma G) \vec{B}_{\perp}+(1+G) \vec{B}_{\|}\right]
$$

(in gaussian unịts)

where $\overrightarrow{\mathrm{B}}_{\perp}$ and $\overrightarrow{\mathrm{B}}_{\|}$are the portions of the magnetic field transverse and parallel to the instantancous velocity of the particle. At the sullie time, the velocity of the particle obeys the Lorentz force equation,

$$
\frac{d \vec{v}}{d t}=\frac{e}{\gamma m c} \vec{v} \times \vec{B}
$$

Using this we may rewrite (1) in terms of the motion of the particle. This is straight forward for the second term; for the first term we proceed as follows:

We assume that the particle moves in the vicinity of a planar reference orbit and is characterized by the Frenet-Serret curvilinear coordinates,

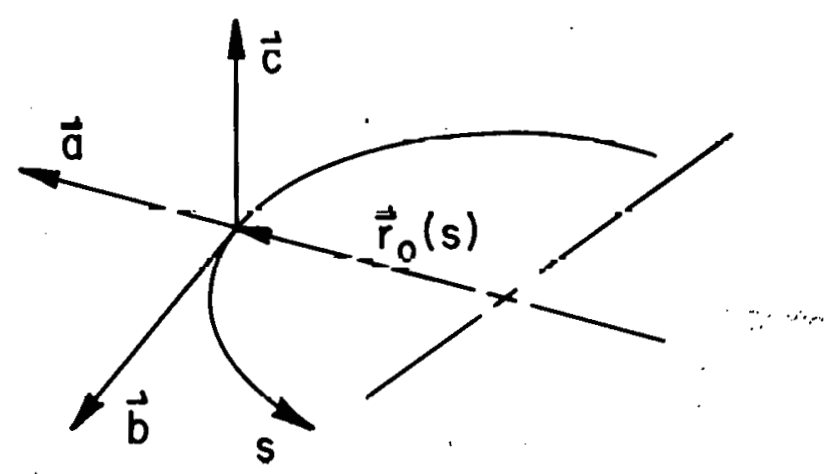

Fig. 1. The Coordinate System... ... 


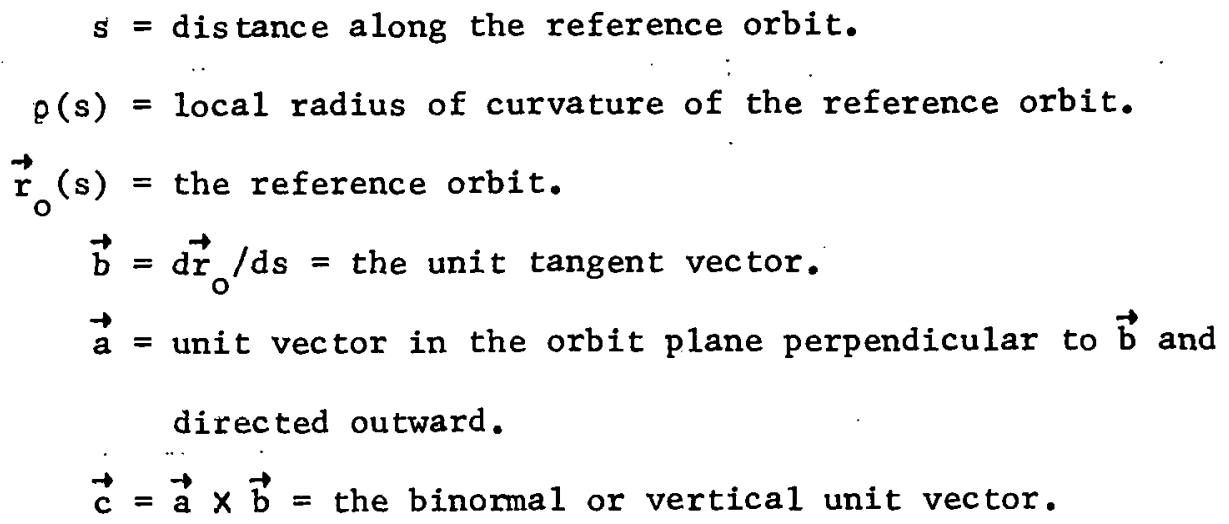

We also have the following relations:

$$
\frac{\overrightarrow{d \vec{a}}}{d s}=\frac{\vec{b}}{\rho} \quad \frac{d \vec{b}}{d s}=\frac{-\vec{a}}{\rho} \quad \frac{d \vec{c}}{d s}=0
$$

An arbitrary point near the reference orbit is characterized by the coordinates $(x, s, z)$ :

$$
\vec{r}=\vec{r}_{0}(s)+x \vec{a}+z_{\vec{c}} \vec{c}
$$

The velocity of a particle is

$$
\vec{v}=\frac{d \vec{r}}{d t}=\frac{d s}{d t} \quad \frac{d \vec{r}}{d s}=\frac{d s}{d t} \quad\left\{x^{\prime} \vec{a}+\left(1+\frac{x}{\rho}\right) \vec{b}+z^{\prime} \vec{c}\right\}
$$

where a prime denotes differentiation by so Keeping terms linear in $s, x, \dot{z}$ and their derivatives we find that

$$
v \equiv|\vec{v}|=\frac{d s}{d t}\left(1+\frac{x}{p}\right)
$$

so that to first order

and

$$
\begin{aligned}
& \vec{v}=v\left(x^{\prime} \vec{a}+\vec{b}+z^{\prime} c\right) \\
& \vec{v}^{\prime}=v\left[\left(x^{\prime \prime}-\frac{1}{\rho}\right) \vec{a}+\frac{x^{\prime}}{\rho} \vec{b}+z^{\prime \prime} \vec{c}\right]
\end{aligned}
$$

Transforming to $s$ as the independent variable while using (2) and (7) we obtain

$\vec{B}_{\perp} \equiv \frac{1}{v^{2}}(\vec{v} \times \vec{B}) \times \vec{v}=B \rho\left(1-\frac{x}{\rho}\right)\left\{\left(x^{\prime \prime}-\frac{1}{\rho}\right) \vec{c}-z \vec{a}+\frac{z^{\prime}}{\rho} \vec{b}\right\}$ 
where $B_{p}=\mathrm{mcv} / \mathrm{e}$ is the magnetic rigidity of the particle. To obtain $B_{\|}$in terms of the particle coordinates, we note that, on the reference orbit, the guide field is just

$$
\vec{B}_{0}=-\frac{(B Q) \vec{c}}{\rho}
$$

Assuming that the longitudinal field $B_{s}$ on the reference orbit is zero, we use Maxwell's curl equation to note that

$$
\frac{\partial}{\partial z} B_{s}=\frac{\partial}{\partial s} B_{z}=-B_{p} \frac{d}{d s}\left(\frac{1}{D}\right)
$$

Thus.

$$
B_{s}=-(B p)<\frac{d}{d s}\left(\frac{1}{\rho}\right)
$$

and to first oxder

$$
\begin{aligned}
& B_{\|}=B_{s}+z^{\prime} B_{o}=-\left[B \rho z \frac{d}{d s}\left(\frac{1}{\rho}\right)+z^{\prime}\left(B_{p}\right) \frac{1}{\rho} \cdot\right] . \\
& \vec{B}_{\|}=-B \rho \frac{d}{d s}\left(\frac{z}{\rho}\right) \vec{b}
\end{aligned}
$$

The spin equation (1) now becomes, to first order in $z$ and $x$,

$$
\begin{aligned}
\frac{d \vec{S}}{d s} & =\vec{S} \times\left\{(1+y G)\left[\left(x^{\prime \prime}-\frac{1}{p}\right) \vec{c}-z^{\prime \prime} \vec{a}+\frac{z^{\prime}}{p} \vec{b}\right]-(1+G)\left(\frac{z}{\rho}\right)^{\prime} \vec{b}\right\} \\
& =\vec{S} \times \vec{F}
\end{aligned}
$$

where

$$
\begin{aligned}
\vec{F}=F_{1} \vec{a}+F_{2} \vec{b}+F_{3} \vec{c}= & -(1+\gamma G) z^{\prime \prime} \vec{a}+\left[(1+\gamma G) \frac{z^{\prime}}{\rho}-(1+G)\left(\frac{z}{\rho}\right)^{\prime}\right] \vec{b} \\
& +(1+\gamma G)\left(x^{\prime \prime}-\frac{1}{\rho}\right) \vec{c}
\end{aligned}
$$


Spelling out the components, $s_{1}=\vec{s} \cdot \vec{a}, s_{2}=\vec{s} \cdot \vec{b}, s_{3}=\vec{s} \cdot \vec{c}$ and noting (3) we find,

$$
\begin{aligned}
& \frac{\mathrm{dS}}{\mathrm{ds}}=\mathrm{s}_{2}\left[\frac{1}{\mathrm{p}}+\mathrm{F}_{3}\right]-\mathrm{s}_{3} \mathrm{~F}_{2} \\
& \frac{\mathrm{dS}}{\mathrm{ds}}=-\mathrm{S}_{1}\left[\frac{1}{\rho}+\mathrm{F}_{3}\right]+\mathrm{s}_{3} \mathrm{~F}_{1} \\
& \frac{\mathrm{dS}}{\mathrm{ds}}=\mathrm{s}_{1} \mathrm{~F}_{2}-\mathrm{s}_{2} \mathrm{~F}_{1}
\end{aligned}
$$

It is convenient to change the independent variable to the turning angle of the reference orbit

$$
\theta=\int_{j 0}^{s} \frac{\mathrm{ds}}{\rho}
$$

since in the field free sections $\theta$ and the spin vector both remain fixed. With these changes we find,

$$
\begin{aligned}
& \frac{\mathrm{dS}}{\mathrm{d} \theta}=-\varkappa \mathrm{S}_{2}-\mathrm{rs}_{3} \quad \text { (a) } \\
& \frac{\mathrm{dS}}{\mathrm{d} \theta}=x \mathrm{~S}_{1}-\mathrm{t \textrm {S } _ { 3 }} \text { (b) } \\
& \frac{\mathrm{dS}}{\mathrm{d} \theta}=r \mathrm{~S}_{1}+\mathrm{ts}_{2} \quad \text { (c) }
\end{aligned}
$$

where

$$
\begin{aligned}
& x \equiv-\left(1+p F_{3}\right)=\gamma G-(1+\gamma G) p x^{\prime \prime} \simeq \gamma G \quad(a)^{*} \\
& r \equiv \rho F_{2}=(1+\gamma G) z^{\prime}-p(1+G)\left(\frac{z}{p}\right)^{\prime} \quad \text { (b) } \\
& t \equiv-\rho F_{1}=(1+\gamma G) \rho z^{\prime \prime}
\end{aligned}
$$

*Note: We let $x=\gamma G$ since the second term is small and averages to zero. 
In the absence of transverse motion $r=t=0$, thus we obtain to lowest order:

$$
\begin{aligned}
& \mathrm{s}_{1}=\sin \alpha \cos \int \gamma G \mathrm{~d} \theta \\
& \mathrm{s}_{2}=\sin \alpha \sin \int \gamma G \mathrm{~d} \theta \\
& \mathrm{s}_{3}=\cos \alpha
\end{aligned}
$$

where $\vec{S}$ has been implicitly normalized by setting $\cos \alpha=s_{3}$, the vertical component of the spin. This motion is simply a precession axound the $z$ axts with a frequency of ye.

Using this solution in the presence of transverse motion, the lowest order change in $\cos \alpha$ is given by:

$$
\frac{\mathrm{d}}{\mathrm{d} \theta}(\cos \alpha)=\sin \alpha\left\{r \cos \int \gamma \mathrm{Gd} \theta+t \sin \hat{i}_{j} \gamma \mathrm{Gd} \theta\right\}
$$

If $r$ and $t$ possess components of frequency $\gamma G$, the above equation yields a secular increase or decrease in the polarization angle $\alpha$. If the accelerator field is "perfect" and is periodic with P periods per circumference, $z$ and therefore $r$ and $t$ will contaln components of frequency $k P \pm v$, where $v$ is the betatron oscillation frequency and $k$ is any integer; thus resonance occurs at energies where $\gamma G=k P \pm v$.

In the presence of imperfection the $P$ periodicity is broken $(P=1)$ which, of course, ylelds resonances at $\gamma G=k \pm v$. But more importantly, the errors in the guide fleld induce errors in the equilibrium orbit that necessarily contaln all Integral Fourier components (since the errors are repeated each cycle or every $2 \pi$ of turning angle); thus, resonance also occurs when $\gamma G=k$ : (A detailed discussion of the relative sizes of each of these three types of resonances. will be left to section V). 
If a resonance condition is satisfied, the subsequent change in $\alpha$ will not be smal1. This means our neglect of the second terms in (17a) and (17b) is not justified, since without these terms the length of the spin vector is not conserved. So to examine in detail the behavior at or near resonance, we must consider the full set of 3 equations (17). Let us first, however, put these in a more convenient form.

We introduce the complex quantities,

$$
\begin{aligned}
& s_{+}=s_{1}+i s_{2} \\
& s_{-}=s_{1}-i s_{2}
\end{aligned}
$$

and the function $\zeta$,

$$
\zeta(\theta)=-t-i r
$$

With these changes our three spin equations become

$$
\begin{aligned}
& \frac{d S_{+}}{d \theta}=i \sim S_{+}+\frac{1}{2} \zeta^{*} S_{3} \quad(a) \\
& \frac{d S_{-}}{d \theta}=-i \wedge s_{-}-\frac{i}{2} \zeta s_{3} \quad \text { (b) } \\
& \frac{d S_{3}}{d \theta}=i\left(\zeta s_{+}-\zeta^{*} s_{-}\right)
\end{aligned}
$$

where $\zeta(\theta)=-(1+\gamma G)\left(z^{\prime \prime}+1 z^{\prime}\right)+1_{p}(1+G)(z / \beta)^{\prime}$.

and $x(\theta)=G_{\gamma}(\theta)$,

To recapitulate, if $\zeta$ contains a Fourier component $\varepsilon e^{-i{ }_{0} \theta}$, resonance occurs when $x \equiv \gamma G=x_{0}$. On the other hand, the set of harmonics which comprise $\zeta$ are given by:

$$
\begin{aligned}
x_{0} & =1: P \pm \nu & & \text { "intrinsic harmonics" } \\
& =k \pm v \text { or } \mathrm{k} & & \text { "imperfection harmonics" }
\end{aligned}
$$


and the Fourier amplitude, $\epsilon$, for a particular harmonic, $x_{0}$, is given by:

$$
\begin{aligned}
\epsilon & =\frac{1}{2 \pi} \int_{0}^{2 \pi} \zeta(\theta) e^{i x_{0} \theta} d \theta \\
& =\frac{1}{2 \pi} \oint \frac{\zeta(s)}{\rho(s)} e^{i x_{0} \theta(s)} d s
\end{aligned}
$$

We leave the calculation of $\varepsilon$ to section $V$ while in the next section we introduce the spinor formulation of equations (23) and present some specific solutions.

III. QUANTUM MECHANICAL FORMULATION

\section{A) The Spinor Equation}

The easiest way to solve equations (23) is by quantum meahanical techniques. By the correspondence principle, the expectation values of quantum mechanical operators satisfy the classical equations of motion of the corresponding variables. Therefore, if we interpret $S_{+}, s_{-}, S_{3}$ as the expectation values of the quantum mechanical operators $\sigma_{+}, \sigma_{-}, \sigma_{3}$; and $1 f$ we solve the Schrodinger equation for a suitable hamiltonian, we have solved the classical equations. 9

We suppose the spin is $\frac{1}{2}$ so that we may deal with 2 component spinors. This is, of course, physically exact if we are dealing with spin $\frac{1}{2}$ particles (protons and electrons). However, even if the actual particle is, say, a deuteron with spin 1 (3 spin states), the classical equations are the same (see Bargman et.al.) ${ }^{8}$, and our solution for the classical equations is still valid, although we do not treat the three spin states properly. 
Taking $\theta$ as our time variable, we look for a Hamiltonian with which,

$$
\frac{\mathrm{d} \psi}{\mathrm{d} \theta}=\frac{i}{2} \mathrm{H} \psi
$$

where $\psi$ is a two component spinor and

$$
s_{i}=\psi^{\dagger} \sigma_{i} \psi
$$

The $\sigma_{i}$ 's are the Pauli matrices, and we recall that:

$$
\begin{aligned}
& \sigma_{x}=\left(\begin{array}{ll}
0 & 1 \\
1 & 0
\end{array}\right) \quad \sigma_{y}=\left(\begin{array}{cc}
0 & -i \\
i & 0
\end{array}\right) \quad \sigma_{z}=\left(\begin{array}{rr}
1 & 0 \\
0 & -1
\end{array}\right) \\
& \sigma_{+}=\frac{1}{2}\left(\sigma_{x}+i \sigma_{y}\right) \quad\left[\sigma_{i}, \sigma_{j}\right]=2 i \varepsilon_{1 j k} \sigma_{k} \\
& \sigma_{-}=\frac{1}{2}\left(\sigma_{x}-i \sigma_{y}\right) \quad\left[\sigma_{ \pm}, \sigma_{z}\right]=\mp 2 \sigma_{ \pm} \\
& {\left[\sigma_{+}, \sigma_{-}\right]=\sigma_{z}}
\end{aligned}
$$

From (27) we find that

$$
\frac{\mathrm{dS}}{\mathrm{d} \theta}=\frac{i}{2} \psi^{i}\left[\sigma_{i}, \mathrm{H}\right] \psi
$$

and one can quickly verify that if we set

$$
\mathrm{H}=\left(\begin{array}{cc}
-x & \zeta \\
5 & x
\end{array}\right)
$$

or alternatively

$$
\mathrm{H}=-n \sigma_{\mathrm{z}}+\zeta \sigma_{+}+\zeta * \sigma_{-}
$$

we obtain the set of equations (23). To restate the problem, we now must solve the time dependent Schrodinger equation (27) with the time dependent Hamiltonian given above.

In the acceleration process $u=\gamma G$ increases with time (or $\theta$ ) and passes through a resonance value $\chi_{0}=\mathrm{kP} \pm \nu$ or $\mathrm{k}$. In order to analyze the effect of a particular resonance we will assume that the resonances are well separated so that each Fourier component of $\zeta$ can be treated separately. In particular we set $\zeta=\varepsilon e^{-i x_{0} \theta}$, 
and from equations (27) and (31) we obtain

$$
\frac{d \psi}{d \theta}=\frac{1}{2}\left(\begin{array}{cc}
-\ddot{x} & \varepsilon e^{-i x_{0} \theta} \\
\varepsilon e^{* i n_{0} \theta} & x
\end{array}\right) \psi
$$

Let us now examine some speciffc solutions to equation (32).

\section{B) Solutions to the Spinor Equation}

1. $\left(x-x_{0}\right)=$ constant. The solution of the spin equations for a beam circulating at constant energy, $x=$ a conotant, is useful In understanding the spin behavior in the neighhorhood of a rcoonance.

Transform Eq. (32) to a new rotating coordinate system by,

$$
\psi=e^{-\frac{i}{2} n_{0} \theta \sigma} z \varphi
$$

We then find

$$
\frac{d \psi}{d \theta}=\frac{1}{2}\left(\begin{array}{rr}
-\delta & \varepsilon \\
\varepsilon & \delta
\end{array}\right) \text { ip }
$$

where $\delta \equiv \mathfrak{x}-\mathfrak{x}_{0}$, and we have let $\varepsilon$ be real and positive for convenience. We diagonalize and obtain the following normal modes:

$$
\varphi_{+}(\delta)=\left(\begin{array}{c}
\sqrt{\frac{\lambda+\delta}{2 \lambda}} \\
-\sqrt{\frac{\lambda-\delta}{2 \lambda}}
\end{array}\right) e^{-\frac{i}{2} \lambda \theta}, \varphi_{-}(\delta)=\left(\begin{array}{c}
\sqrt{\frac{\lambda-\delta}{2 \lambda}} \\
\sqrt{\frac{\lambda+\delta}{2 \lambda}}
\end{array}\right) e^{\frac{i}{2} \lambda \theta}
$$

where $\lambda=+\sqrt{\varepsilon^{2}+\delta^{2}}$.

The general solution is given by

with

$$
\begin{gathered}
\varphi-c_{+}++c_{-}+c_{-}, \\
\left|c_{+}\right|^{2}+\left|c_{-}\right|^{2}=1
\end{gathered}
$$

We are interested in the vertical projection of the spin, which is 


$$
s_{z}=\varphi^{\dagger} \sigma_{z} \varphi=\left(\left|C_{+}\right|^{2}-\left|C_{-}\right|^{2}\right) \frac{\delta}{\lambda}+2\left|C_{+}\right|\left|c_{-}\right| \frac{\varepsilon}{\lambda} \cos (\lambda \theta+\Delta)
$$

where $\Delta$ is just the relative phase of $C_{+}$and $C_{-}$. The equation just solved is a single particle equation. Since $\Delta$ is different for different particles, the $2 \mathrm{n} d$ term in $\mathrm{Eq}$. (37) yields a spread in $\mathrm{S}_{z}$; however, if we are interested in an average over particles, the second term does not contribute. In the subsequent discussion we will drop the 2 nd term in Eq. (37) and all values will be average values.

Notice that for pure states $S_{z}= \pm \delta / \lambda$ and $f \mathrm{cr} \delta \gg \varepsilon, S_{z} \rightarrow \pm 1$, a spin up state and a spin down state as one would expect. But if we begin with $S_{z}=+1$ and $\delta=-\infty$ and allow $\delta$ to vary only slowly (adiabatically), then $\varphi, \varphi_{+}$and $\varphi_{-}$all obey the same equation so that $C_{+}$and $C_{-}$are invariant. As we approach the resonance, $S_{z} \rightarrow \delta / \lambda$; and as we pass through the resonance (adiabatically), $\delta$ changes sign so that as $\delta \rightarrow+\infty, S_{z} \rightarrow-1$. This spin flip is just the so called "fast adiabatic passage through resonance" well known from the theory and practice of Nuclear Magnetic Resonance.

The salient point here is that in spite of the apparent depolarization $\left(S_{z}=\delta / \lambda\right)$ near the resonance, if we vary $\delta$ adiabatically, the depolarization is only apparent since the spin will reorient itself as $\delta \rightarrow \pm \infty$.

2. $x-x_{0}=a_{\theta}$. Equation (32) has also been solved exactly ${ }^{1}$ for this case. Although Froissart and Stora obtain the exact solution in terms of confluent hypergeometric functions, the resuit usually quoted is obtained from the asymptotic form. Let us just examine this asymptotic region. Transforn Fq. (32) to the "interaction representation" by 
the transformation:

$$
\psi=\mathrm{e}^{-1 / 2 \int_{0}^{\theta} x d \theta \sigma_{z}}
$$

then

$$
\frac{d \xi}{d \theta}=\frac{i}{2}\left(\begin{array}{cc}
0 & \varepsilon e^{i X} \\
\varepsilon e^{-i x} & 0
\end{array}\right) \xi
$$

where

$$
X=\int_{0}^{\theta}\left(x-x_{0}\right) d \theta=\alpha \theta^{2} / 2 .
$$

Nuw let $\xi=\left(\begin{array}{l}f \\ g\end{array}\right)$, and L'q. (39) becomes

$$
\frac{\mathrm{d}^{2} \mathrm{f}}{\mathrm{d} \theta^{2}}-i \alpha \theta \frac{\mathrm{df}}{\mathrm{d} \theta}+\frac{\varepsilon^{2}}{4} \mathrm{f}=0
$$

It is easy to show that $S_{z}$ is now given by

$$
S_{z}=|f|^{2}-|g|^{2}=2|f|^{2}-1
$$

The behavior near $\theta=0$ is just harmonic as one can see by dropping the middle term in Eq. (41). On the other hand the behavior as $\theta \rightarrow \infty$ is dominated by the 2 nd two. terms so we have

$$
-i \alpha \theta \frac{d t}{d \theta}+\frac{\varepsilon^{2}}{4} f \simeq 0 \text { as } \theta \rightarrow \infty .
$$

This can be readily integrated to yield

$$
\frac{f(\infty)}{f(-\infty)}-\exp \left(\frac{-1 \varepsilon^{2}}{4 \alpha} \quad \int_{-\infty}^{\infty} \frac{d \theta}{\theta}\right)
$$

If we select the physically meaningful path around the pole in Eq. (44) $(|t|<1)$, then we obtain

$$
\left|\frac{f(\infty)}{f(-\infty)}\right|=e^{-\pi \varepsilon^{2} / 4 \alpha}
$$


So that if $S_{z}(-\infty)=1$, then from Eq. (42)

$$
S_{z}(\infty)=2 e^{-\pi \varepsilon^{2} / 2 \alpha}-1
$$

This is the result of Froissart and Stora which they obtained by using asymtotic forms of the exact solution. Equation (46) is particularly useful when calculating depolarization due to normal, linear acceleration provided the resonances are well. separated. It is also useful for determining the acceptable $\varepsilon^{2} / \alpha$ for an adiabatic passage through resonance; however, in applying the above formula one must be sure the asymptotic form is applicable.

Let us anticipate the results of section $V$ by referring to Fj.g. 2 which presents calculations of $\varepsilon$ for all the resonances in the Brookhaven AGS. Also presented in the figure is the effect of these resonances calculated with the results of Froissart and Stora, Eq. (46), using the normal AGS acceleration rate $(\alpha=G d \gamma / d t)$. One can clearly see from this figure that a polarized proton beam will quickly become depolarized unless something is done to counteract the effect of the resonances. In the next section we describe a cure for the intrinsic resonances.

IV. THE RESONANCE JUMP

The standard method ${ }^{5}$ for dealing with the intrinsic resonances is to "jump" them by changing the tune, $\nu$, abruptly as $x$ approaches a resonance, $x_{0}=k P \pm v$, during normal acceleration. This change in $v$ increases $\alpha$ in the neighborhood of the resonance, and qualitatively, using Eq. (46), one would expect much improvement if $\alpha$ is made sufficiently large. However, Eq. (46) is not strictly applicable 
In this case, and we expect the size of the resonance jump to be a significant factor also. To see this quantitatively we w1ll consider the following realistic model:

1. Let $x_{0}$ be an isolated resonance.

2. Let $x-x_{0}$ vary in a three step process,

$$
x-x_{0}=\left\{\begin{array}{c}
-\infty \text { to }-\delta \text {, very slowly, adiabatically } \\
-\delta \text { to } \delta, \text { fast jump, } \frac{d\left(x-x_{0}\right)}{d \theta}=\alpha \\
\delta \text { to } \infty, \text { again adiabatically. }
\end{array}\right.
$$

A. The tails of the resonance, $x-x_{0} \rightarrow-\infty$ to $-\delta$ and $\delta$ to $\infty$ adiabatically. The solution tn spin equation with adiablic variation has been given in Eq. (35). Let us consider the initial condition $S_{z}(-\infty)=+1$. This is equivalent to

$$
\varphi_{-}(-\infty)=\left(\begin{array}{l}
1 \\
0
\end{array}\right)
$$

which adlabatically transforms to

$$
\Psi_{-}(-\delta)=\left(\begin{array}{c}
\sqrt{\frac{\lambda+\delta}{2 \delta}} \\
\sqrt{\frac{\lambda-\delta}{2 \lambda}}
\end{array}\right)
$$

The spin up spinor on the other side of resonance is given by

$$
\varphi_{+}(\delta)=\left(\begin{array}{l}
\sqrt{\frac{\lambda+\delta}{2 \lambda}} \\
-\sqrt{\frac{\lambda-\delta}{2 \lambda}}
\end{array}\right)
$$

and this adiabatically transforms to

$$
\varphi_{+}(+\infty)=\left(\begin{array}{l}
1 \\
0
\end{array}\right)
$$


and similarly for $\varphi_{-}(\delta)$.

A general spinor at $\delta$ is, of course,

$$
\varphi(\delta)=C_{+} \varphi_{+}(\delta)+C_{-} \varphi_{-}(\delta)
$$

so as we vary $\delta \rightarrow+\infty$ adiabatically, we find,

$$
\varphi(+\infty)=\left(\begin{array}{l}
C_{+} \\
C_{-}
\end{array}\right)
$$

We can calculate $S_{z}(+\infty)$ by

$$
\mathrm{S}_{z}(+\infty)=\varphi^{\dagger}(+\infty) \sigma_{z} \varphi(+\infty)=2\left|\mathrm{C}_{+}\right|^{2}-\ddot{1}
$$

All that is necessary to complete the calculation is to calculate $\left|c_{+}\right|$ after the jump.

B. The resonance jump, $x-x_{0} \rightarrow-\delta$ to $\delta, \theta \rightarrow-\delta / \alpha$ to $\delta / \alpha$. In the region from $-\delta$ to $\delta$ we let $x-x_{0}=\alpha \theta$. Since we know the exact solutions for this problem, 1 we could simply use the results of Froissart and Stora integrated from $-\delta / \alpha$ to $\delta / \alpha$. However, since the jump is a "fast" jump $\left(\pi \varepsilon^{2} / \alpha<<1\right)$, and since we seek simple formulae, we will use a scattering matrix approach instead. We introduce the scattering operator, $U$, in the interaction representation by

$$
\xi(\theta)=U\left(\theta_{,} \theta_{0}\right) \xi\left(\theta_{0}\right)
$$

then we find from Eq. (39)

$$
\frac{d U}{d \theta}=\frac{i}{2} V U, \quad V=\left(\begin{array}{cc}
0 & \varepsilon e^{i \alpha \theta^{2} / 2} \\
\varepsilon e^{-i \alpha \theta^{2} / 2} & 0
\end{array}\right)
$$

where $U$ is a $2 \times 2$ unitary unimodular (SU2) matrix. If we keep the first 2 terms In the standard iterative solution of Eq. (55), we obtain

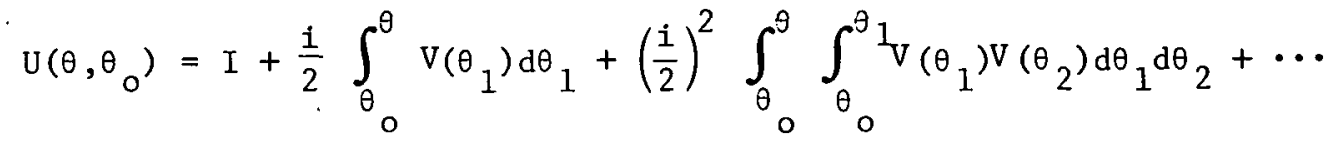


where $I$ is the identity. Written explicity;

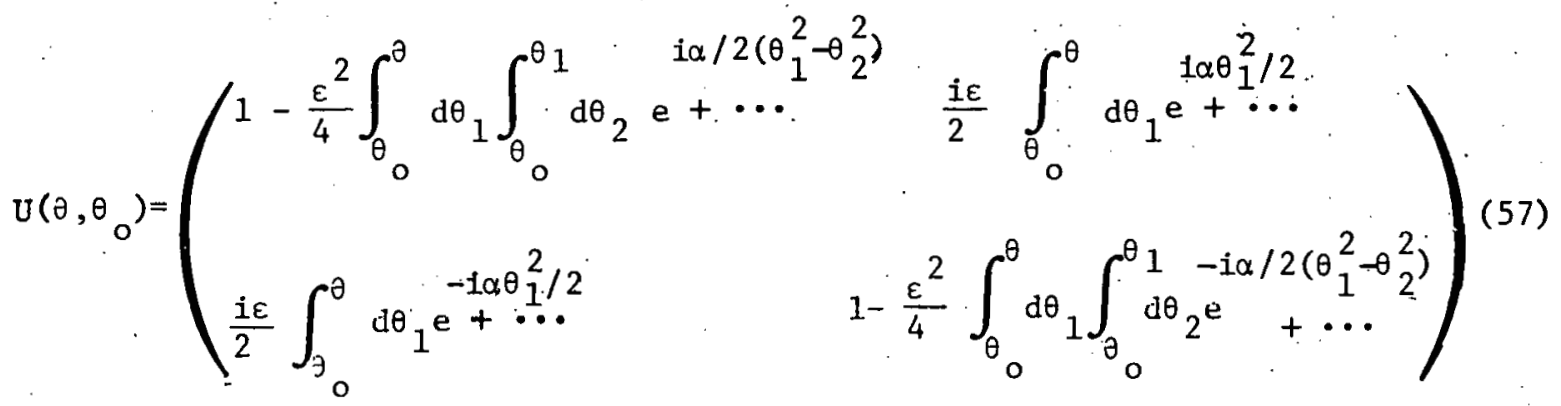

We know the spinor at $-\delta$; to calculate the spinor at $\delta$ after scattering through the resonance, simply transform $U$ to the came coordinate system as $\varphi_{-}(-\delta)$. Then our spinor at $\delta$ is given by

$$
\varphi(\delta)=e^{-i\left(\delta^{2} / 4 \alpha\right) \sigma_{z}} \mathrm{u}(\delta / \alpha,-\delta / \alpha) e^{i\left(\delta^{2} / 4 \alpha\right) \sigma_{z}} \varphi_{-}(-\delta)
$$

Now the calculation of $\mathrm{C}_{+}$is just a projection onto the basis spinor at $\delta$;

$$
c_{+}=\varphi_{+}^{+}(\delta) p(\delta)
$$

or using (48), (49), and (57)

$$
c_{+}=1-\frac{\varepsilon^{2}}{4} \frac{L_{1} 1_{1}^{*}}{2} 8 / \lambda+i \frac{\varepsilon^{2}}{l_{1}}\left(1_{1}-1_{1}^{*}\right)
$$

where

$$
I_{1}=e^{-i \delta^{2} / 2 \alpha} \int_{-i / \alpha}^{\delta / \alpha} e^{i \alpha \theta^{2} / 2} d \theta
$$

( $\mathrm{I}_{1}$ is a combination of Fresnel integra1s)

It is instructive at this point to compare this result to that or Frolssart and stora, In that case $f$ plays the role of $C_{+}$,

$$
f=e^{-\pi \varepsilon^{2} / 4 \alpha} \simeq 1-\frac{\pi \varepsilon^{2}}{4 \alpha} \text { for "fast" passage. }
$$


If we let $\delta \rightarrow \infty$ in Eq. (60), the last term goes to zero, $\delta / \lambda+1$ and $\mathrm{I}_{1} \mathrm{I}_{1}^{*} \rightarrow(2 \pi / \alpha)$, thus we recover Eq. (62).

On the other hand we can also consider the case when $\alpha \rightarrow \infty$, an instantaneous crossing. In this case $\mathrm{C}_{+} \rightarrow \delta / \lambda$ so that

$$
S_{z} \rightarrow \frac{2 \delta^{2}}{\lambda^{2}}-1=\frac{\delta^{2}-\varepsilon^{2}}{\delta^{2}+\varepsilon^{2}} \quad \text { as } \alpha \rightarrow \infty
$$

So we are left with substantial depolarization even when the resonance is crossed instantly. We will soon see that this can be the dominant effect even when $\alpha$ is finite. It is easy to understand the preceeding effect by examining the spinors in (48). and (49): Since $c_{+}$in the above case is the overlap of the eigenfunctions at $\delta$ and $-\delta$, the depolarization is due to their mismatch. The spinors in (48). and (49) are out of phase by $e^{i \pi \sigma_{z}}$ so their overlap is not unity. This phase difference is due to the reversal of the effective field in which the spin is precessing. One might say our "top" has found itself suddenly with a torque of the opposite sign. The point, however, of this exercise is recognize the relative importance of $\delta$ and $\varepsilon$, e.g., when $\delta=\varepsilon$ we obtain complete depolarization.

ReLuraing to Eq. (60) notice that the argument of the exponential in the integral is always quite small. For example, if we use the parameters for the proposed polarization of the AGS, 10 then $\delta=0.125$, $\alpha=0.0597$, so $\left(\delta^{2} / 2 \alpha\right)=0.131$. So we can expand the exponentials to evaluate $I_{1}$.

$$
\begin{aligned}
& I_{1} \simeq 2\left(1-i \frac{\delta^{2}}{2 \alpha}\right)\left(\frac{\delta}{\alpha}+\frac{i \alpha}{6}(\delta / \alpha)^{3}\right) \\
& I_{1} \simeq 2\left(\delta / \alpha-\frac{i \delta^{3}}{3 \alpha^{2}}\right)+\text { higher order terms. }
\end{aligned}
$$


with this we find

$$
c_{+} \simeq\left(1-\frac{\varepsilon^{2} \delta^{2}}{6 \alpha^{2}}\right) \frac{\delta}{\lambda}
$$

which together with Eq. (53) yields

$$
S_{z} \simeq \frac{\delta^{2}-\varepsilon^{2}}{\delta^{2}+\varepsilon^{2}}-\frac{2 \varepsilon^{2} \delta^{2}}{3 \alpha^{2}} \frac{\delta^{2}}{\left(\delta^{2}+\varepsilon^{2}\right)}
$$

restrictions: $\delta^{2} / 2 \alpha \ll 1$, valid to order $\left(\varepsilon^{2} / \alpha\right)$.

Equation (66) is the final rcsult for the depolarization due to a resuladuce fump, the dominant offoct io not leyeuleul on the rate of passage through the resonance, but rather on the size of the resnnance jump as compared to the width, $\varepsilon$, of the resonance. Consider, for example, the resonance at $x=\nu$ in the AGS. From Table 1 we spp that the total depolarization calculated with Eq. (66) is 0.969. However, the first term in Eq. (66) yields $P / P_{0}=0.970$ while the 2 nd term gives only an additional -0.001 . This does not mean that the rate of passage is unimportant since the dependence is $1 / \alpha^{2}$. However, it is clear in the above example that an increase in $\alpha$ has little effect on the depolarization. The alove lesult 1.s primarily for resonances which are quite strong. $\left(e^{-\pi \varepsilon^{2} / 4 \alpha} \simeq 0.005, \alpha=G \dot{d} \gamma / d t\right)$ so that the statement of adiabatic approach and departure applies. If this condition is not satisfled, Eq. (66) should be considered a lower hound on $\mathrm{S}_{z}$.

In all the solutions given in the previous two sections the critical parameter is $\varepsilon$, the strength or width of a resonance. In the next section we develope an expression suitable for the numerical calculation of $\varepsilon$ in any accelerator. 
Table I

The Resonance Jumps

Depolarization Due to AGS Intrinsic Resonances Magnet full field risetime $=2 \mu \mathrm{sec}(4 \pi / 3$ radians $) . \delta=0.125, \alpha \equiv \mathrm{d} \nu / \mathrm{d} \theta=0.0597, \nu=8.75$.

\begin{tabular}{|c|c|c|c|}
\hline $\begin{array}{l}G_{\text {res }}= \\
\mathrm{kP} \pm \nu\end{array}$ & rres & $\varepsilon$ & $\begin{array}{l}\text { Polarization } \\
\text { P/Po } \\
\text { Eq. (66) }\end{array}$ \\
\hline $12-v$ & 1.81 & 0.0054 & 0.996 \\
\hline $0+v$ & .4 .88 & 0.0154 & 0.969 \\
\hline $24-v$ & 8.51 & 0.0006 & 1.000 \\
\hline $12+v$ & 11.57 & 0.0054 & 0.996 \\
\hline $36-v$ & 15.20 & 0.0137 & 0.975 \\
\hline $24+v$ & 18.26 & 0.0010 & 1.000 \\
\hline $48-v$ & 21.89 & 0.0015 & 1.000 \\
\hline $36+v$ & 24.96 & 0.0266 & 0.911 \\
\hline $60-v$ & 28.86 & 0.1576 & $-\cdots *$ \\
\hline $48+v$ & 31.65 & 0.0023 & 0.999 \\
\hline \multicolumn{4}{|c|}{ Resultant polarization after acceleration up to: } \\
\hline $36+v$ & \multicolumn{2}{|c|}{24.96} & 0.853 \\
\hline
\end{tabular}

V. THE DEPOLARIZATION COEFFICIENT, $\varepsilon$

A. The Types of Resonance

Before we calculate $\&$ for a particular resonance, let us first examine the three different types of resonances which are possible:

1. $n_{0}=\mathrm{kP} \pm v$, those which are due to the betatron oscillations of the "perfect" machine.

2. $x_{0}=k \pm v$, those which are caused by the gradient errors present in an actual machine. 
3. $x_{0}=k$, those which are caused by errors in magnet alignment or field.

First we write the $z$ motion as:

$$
z=z_{e}+z_{\beta}
$$

where $z_{e}$ is the displacement of the equilibrium orbit due to errors of type (3), and $z_{\beta}$ is the vertical betatron oscillation of the actual machine. These obey

$$
\begin{aligned}
& z_{\beta}^{\prime \prime}+K(s) z_{\beta}=0 \\
& z_{e}^{\prime \prime}+K(s) z_{e}=F(s)
\end{aligned}
$$

where $K(s)=B^{\prime} / B p$ is the actual focusing function (including errors), and $F(s)$ is a measure of the field error on ideal equilibrium orbit. Solving equation ( $2 a$ ) we find for the betatron oscillations 11

$$
z_{\beta}=\frac{1}{2} \sqrt{\varepsilon \beta(\theta)}\left[e^{-i[\forall \theta-x(\theta)]}+c \cdot c \cdot\right]
$$

where $v \theta-x(\theta)=v \int_{0}^{s} \frac{d s}{v \beta}$ is the betatron oscillation phase, $x(\theta) \equiv v \int(1 / \rho-1 / \psi \beta)$ ds, $\beta$ is the Courant-Snyder amplitude function, and $\varepsilon$ is the emittance.

Now, if we select any one point on the circumference, say $\theta_{0}, x\left(\theta_{0}\right)$ is just a phase shift, and as the particle passes $\theta_{0}$ on sucressive revolutions, we see it trace a vertical oscillation of amplitude $\sqrt{\varepsilon \beta\left(\theta_{a}\right)}$ and frequency $\vee$. So in the ideal machine since $\sqrt{\beta}$ has periodicity $P$, $z_{\beta}$ contains frequencies $k P \pm v$. On the other hand, if we allow gradient crrors to be presene, our tocusing function $K$ and therefore $\beta$ are only strictly periodic with period of one cycle $(P=1)$. These errors shift the tune.slightly and also introduce integer frequencies into $\beta$. Thus, $z_{B}$ will contain frequencies $k \pm v$. 
To see the effect of errors of type (3) we note that $F(s)$ and, thus, $z_{e}$ in equation $(68 \mathrm{~b})$ contains all integer Fourier frequencies. One finds upon solving this equation that the size of particular component of $z_{e}$ depends critically on the proximity of its frequency to $\nu(z)$ is largest when $k$ is near $v){ }^{11}$ Since these equilibrium orbit distortions may be rather large in any particular accelerator, we must consider their effect on depolarization. This is not difficult since within any given magnet if we neglect 1 ts small misalignment; $z_{e}$ also obeys the homogeneous equation (68a), and we may therefore write

$$
\begin{gathered}
z^{\prime \prime}+K z=0 \\
\text { (within a magnet) }
\end{gathered}
$$

Since $\zeta$ and thus $\varepsilon$ depends critically on the magnitude of $z$ oscillating at a particular frequency $x_{0}$, one can order the sizes of the various resonances as $k P \pm \nu>k>k \pm v$, while remembering that for $k \sim v$, the integer imperfection resonances may be as large as the intrinsic resonances.

\section{B. Calculation of $\varepsilon$}

The Fourier amplitude of $\zeta$ which determines the depolarization at a particular resonance $x_{0}$ is given by

$$
\varepsilon=\frac{1}{2 \pi} \oint \frac{\zeta(s)}{\rho\left(s^{i}\right)} \mathrm{e}^{i \varkappa_{0} \theta(s)} \mathrm{ds}
$$

As we have seen, this is essentially the width of the resonance. In most accelerators, the lattice consists of discrete sectors with locally constant field and gradient.separated by field free sections. It is, therefore, convenient to break up the integral into contributions 
from separate magnets as follows:

$$
\varepsilon_{m}=\int_{s_{1}}^{\substack{\varepsilon=\Sigma \\ \text { lattice }}} \frac{\zeta(s)}{\rho(s)} e^{i \varkappa_{0} \theta(s)} d s
$$

where $s_{1}$ and $s_{2}$ lie in the field free region just outside the magnet ends, i.e.,

$$
\begin{aligned}
& K\left(s_{1}\right)=K\left(s_{2}\right)=0 \\
& \frac{1}{\rho}\left(s_{1}\right)=\frac{1}{\rho}\left(s_{2}\right)=0
\end{aligned}
$$

From Ëq. (24) our expression for $\varepsilon_{\mathrm{m}}$ is now,

$$
\epsilon_{\mathrm{m}}=\frac{-1}{2 \pi} \int_{s_{1}}^{s_{2}}\left\{\left(1+x_{0}\right) \cdot\left(z^{\prime \prime}+\frac{i z^{\prime}}{\rho}\right)-i(1+G)\left(\frac{z}{\rho}\right)^{\prime}\right\} e^{i x_{0} \theta} d s
$$

where we have set $\gamma G=\varkappa_{0}$ since we want $\epsilon_{\mathrm{m}}$ at a resonance. We begin by evaluating the two integrals,

$$
\begin{aligned}
& \overline{\mathrm{I}}_{2} \equiv \int \frac{\underline{z}^{\prime}}{\rho} e^{i \kappa_{u} \mathrm{~A}^{\mathrm{d}} \mathrm{d} s} \\
& \mathrm{I}_{3}=\int\left(\frac{\mathrm{z}}{\rho}\right)^{\prime} \mathrm{e}^{i \mu_{0} \theta} \mathrm{ds}
\end{aligned}
$$

Remembering that $1 / p$ is a step function, we can integrate by parts,

$$
I_{2}=\frac{1}{\rho} \int z^{\prime} e^{i \lambda_{0} \theta} d s=\frac{1}{\rho}\left(z_{2} e^{i \lambda_{0} \theta_{2}}-z_{1} e^{j \mu_{0} \theta} 1\right)-\frac{i \mu_{0}}{\rho^{2}} \int_{s_{1}}^{s_{2}} z e^{i \mu_{0} \theta} d s .
$$

and

$$
\begin{aligned}
& I_{3}=\left.\left(\frac{2}{\rho} e^{i \varkappa_{0} \theta}\right)\right|_{s_{1}} ^{s_{2}}-\frac{i \varkappa_{o}}{\rho} \int_{s_{1}}^{s_{2}} z e^{i \varkappa_{0} \theta} d s \\
& I_{3}=-\frac{i \varkappa_{0}}{\rho} \int_{s_{1}}^{s} z e^{i \varkappa_{0} \theta} d s
\end{aligned}
$$


where we write $z_{1}$ for $z\left(s_{1}\right)$, etc.

Our expression for ${ }_{\mathrm{m}}$ now becomes,

$$
\begin{aligned}
& \epsilon_{m}=-\frac{1}{2 \pi}\left\{\left(1+x_{0}\right) \cdot \int_{1}^{2} z^{\prime \prime} e^{i x_{0} \theta} d s+\frac{x_{0}}{\rho^{2}}\left(x_{0}-G\right) \int_{1}^{2} z e^{i x_{0}{ }^{\theta}} d s\right. \\
& \left.+i \frac{\left(1+\mu_{0}\right)}{\rho}\left(z_{2} e^{i \varkappa_{0} \theta_{2}}-z_{1} e^{i \mu_{0} \theta} 1\right)\right\}
\end{aligned}
$$

If we integrate first over the ends of the magnet, the second integral gives no contribution ( $z$ is continuous); however, the first gives a contribution due to the edge focusing of the magnet.

If the magnet has edges at angles $\xi_{1}$ and $\xi_{2}$ to the curvature vector (as, for example, at the ZGS) then at the ends

$$
\mathrm{K}=\frac{1}{p}\left[\xi_{1} \delta\left(s-s_{1}\right)+\xi_{2} \delta\left(s-s_{2}\right)\right]
$$

$\xi$ is taken as positive if the edge produces vertical focusing. (For a rectangular magnet such as those used at the Brookhaven AGS, $\xi=\ell / 2 p$ where $l$ is the magnet length). So the contribution to $\epsilon_{m}$ from the ends is:

$$
+\frac{1}{2 \pi}\left(1+x_{0}\right)\left(\frac{\xi_{1}}{\rho} z_{1} e^{i x_{0} \theta_{1}}+\frac{\xi_{2}}{\rho} z_{2} e^{i u_{0} \theta_{2}}\right)
$$

Using (80) and (70) we have,

$$
\begin{aligned}
\epsilon_{\mathrm{m}} & =\frac{1}{2 \pi}\left\{\left(\frac{x_{0}\left(x_{0}-G\right)}{\rho^{2} \mathrm{~K}}-\left(1+x_{0}\right)\right) \int_{s_{1}}^{s} z^{\prime \prime} \mathrm{e}^{i x_{0} \theta} \mathrm{ds}\right. \\
& \left.+\frac{\left(1+x_{0}\right)\left(\xi_{1}+i\right)}{\rho} z_{1} e^{i x_{0} \rho_{1}}+\frac{\left(1+x_{0}\right)\left(\xi_{2}-i\right)}{\rho} z_{2} e^{i x_{0} \hat{i}}\right\}
\end{aligned}
$$

where we intcgrate now over the interior of the magnet where $\rho$ and $K$ are constant. 
In order to evaluate,

$$
I_{4} \equiv \int_{s_{1}}^{s_{2}} z^{\prime \prime} e^{i \varkappa_{0} \theta}
$$

We integrate successively by parts and.find that,

$$
I_{4}=\left.\left(z^{\prime} e^{i x_{0} \theta}\right)\right|_{s_{1}} ^{s_{2}}-\left.\frac{i x_{0}}{\rho}\left(z e^{i x_{0} \theta}\right)\right|_{s_{1}} ^{s_{2}}+\frac{x_{0}^{2}}{k_{p}^{2}} I_{4}
$$

or

$$
I_{4}=\frac{\left(z_{2}^{\prime}-\frac{i x_{0}}{\rho} z_{2}\right) e^{i x_{0}^{\theta} 2}-\left(z_{1}^{\prime}-\frac{i x_{0}}{\rho} z_{1}\right) e^{i x_{0} \theta_{1}}}{1-x_{0}^{2} / k_{p}^{2}}
$$

However, from accelerator theory we know that, 10

$$
\left(\begin{array}{c}
z_{2} \\
z_{2}^{\prime}
\end{array}\right)=\left(\begin{array}{ll}
\cos \varphi & \frac{\sin \varphi}{\sqrt{k}} \\
-\sqrt{k} \sin \varphi & \cos \varphi
\end{array}\right)\left(\begin{array}{c}
z_{1} \\
z_{1}^{\prime}
\end{array}\right)
$$

where $\varphi \equiv \sqrt{\mathrm{K}}\left(s_{2}-\mathrm{s}_{1}\right)=\sqrt{\mathrm{K}} l, l$ being the length of the magnet. Solving these equations for $z_{2}^{\prime}$ and $z_{1}^{\prime}$ we obtain,

$$
\begin{aligned}
& z_{1}^{\prime}=\frac{\sqrt{k} z_{2}}{\sin \varphi}-\sqrt{k} \operatorname{ctn} \varphi z_{1} \\
& z_{2}^{\prime}=\frac{\sqrt{k} z_{1}}{\sin \varphi}+\sqrt{k} \operatorname{ctn} \varphi z_{2}
\end{aligned}
$$

If we now define

$$
\theta \equiv\left(\theta_{2}-\theta_{1}\right)=\ell / p
$$

and note that

$$
\begin{aligned}
& z_{1} e^{i x \theta} 2=z_{1} e^{i \mu \theta} e^{i x \theta_{1}} \\
& z_{2} e^{i \mu \theta} 1=z_{2} e^{-i x \theta} e^{i n \theta} 2
\end{aligned}
$$


we obtain,

$$
\begin{aligned}
I_{4} & =\frac{k_{0}^{2}}{k_{\rho}^{2}-x_{0}^{2}}\left\{\left(\frac{\sqrt{k}\left(\cos \varphi-e^{i n \theta}\right)}{\sin \varphi}+\frac{i x_{0}}{\rho}\right) z_{1} e^{i \varkappa_{\rho}{ }_{1}}\right. \\
& \left.+\left(\frac{\sqrt{k}\left(\cos \varphi-e^{-i x \theta}\right)}{\sin \varphi}-\frac{i \kappa_{0}}{\rho}\right) z_{2} e^{i x_{0} \theta_{2}}\right\}
\end{aligned}
$$

so if we let

$$
c \equiv \frac{K_{p}^{2}\left(1+u_{0}\right)-x_{0}\left(u_{0}-G\right)}{x_{0}{ }^{2}-K_{p}{ }^{2}}\left\{\frac{\sqrt{K}\left(\cos \varphi-e^{i u_{0} \theta}\right)}{\sin \varphi}+\frac{i x_{0}}{\rho}\right\}
$$

we have

$$
\begin{aligned}
\varepsilon_{m} & =\frac{1}{2 \pi}\left\{\left(c+\frac{\left(1+x_{0}\right)}{\rho}\left(\xi_{1}+i\right)\right) z_{1} e^{i x_{0} \theta_{1}}\right. \\
& \left.+\left(c^{*}+\frac{\left(1+x_{0}\right)}{\rho}\left(\xi_{2}-i\right)\right) z_{2} e^{i x_{0} \theta_{2}}\right\}
\end{aligned}
$$

and $6=\Sigma 6_{\mathfrak{m}} \cdot$

Now for $x_{0}=k P \pm v$, the contribution to the sum from $z_{e}$ will be zero and so we need only evaluate (89) for $z=z_{\beta}$ (see Eq.(69). On the other hand, when $x=k$ then the contribution from $z_{\beta}$ to the sum is zero; so we may evaluate $\epsilon_{m}$ with $z=z_{e}$ Either of these vertical displacements may be evaluated for a given magnet in a given accelerator by using a computer program such as SYNCH or AGS. One can then perform the sum to evaluate $\varepsilon$.

In Fig. 2 we present the results of this section applied to a calculation of the widths of depolarizing resonances in the AGS at Brookhaven National Laboratory. Notice that the intrinsic resonances are significantly larger than most of the imperfection resonances; however, some of the imperfection resonances are large enough to cause substantial depolarization during normal acceleration. The set of imperfection resonances plotted are those 
obtained from a particular random set of magnet errors. For a different set of errors of the same magnitude, we obtain results which are qualitatively the same although different in detail.

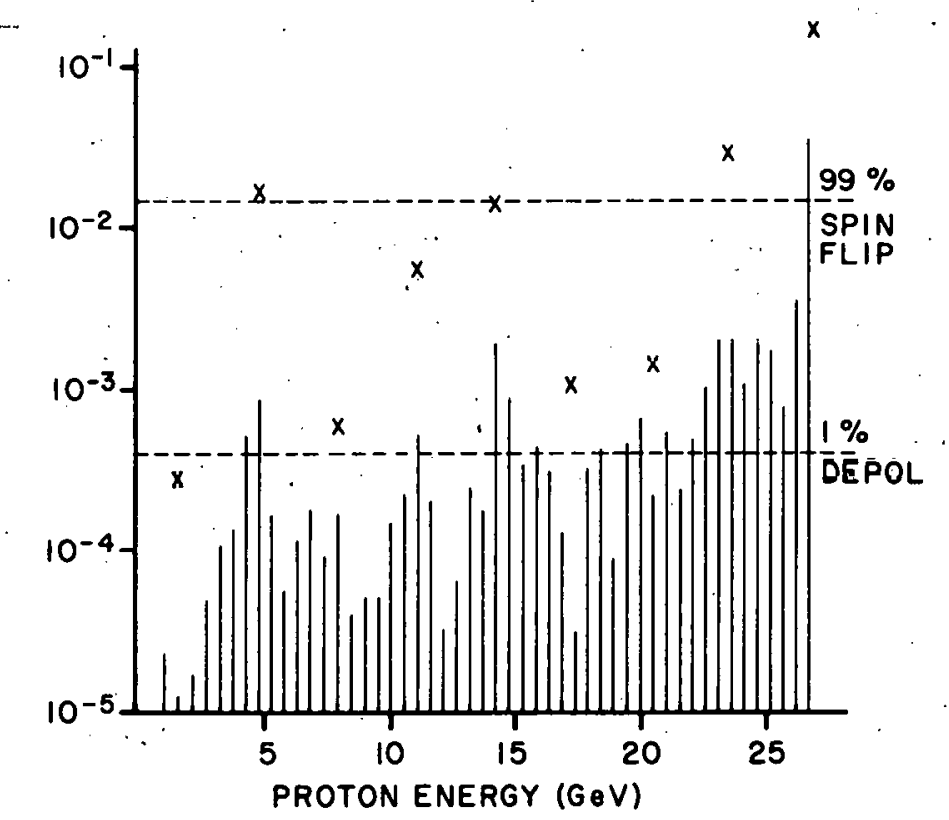

Fig. 2. AGS Resonance Strengths, $\varepsilon$. AGS emittance $=10 \pi / \gamma \mathrm{mr}-\mathrm{mm}$, magnet errors $= \pm 0.1 \mathrm{~mm}, \mathbf{x}$ - intrinsic resonances; $\mid$ - imperfection resonances.

\section{Discussion}

We have presented here a discussion of the theory of depolarizing resonances in proton accelerators. Of the two types of resonances, the Intrinsic resonances are the most troublesome. Table 1. shows the theory of resonance jumps applied to the AGS at Brookhaven National Laboratory. As you can see, for the values of the parameters chosen the total depolarizallun at $26 \mathrm{GeV}$ is only $15 \%$ (for mure detalls of the proposed design see Ref. 9 ). The depolarization due to a resonance jump has also been studied by Turrin. ${ }^{12}$ He used a different model, but his results yield numerical values essentially identical to those in Table 1. 
One of the intrinsic resonances shown in Fig. 2 is so strong that the resonance jump method does not work. In this case it might be possible to traverse the resonance slowly, inducing the beam to flip its spin. But there is a problem with that approach. Even though the $\varepsilon$ shown is quite large, it represents a $95 \%$ betatron amplitude. The beam has a distribution of amplitudes and therefore a distribution of $\varepsilon$ 's. This means that while the spin of the large amplitude particles may flip, the spins of particles at very small or zero amplitude will not. One way to side step this is to provide the beam with a coherent amplitude so that all particles will have a non zero, large $\varepsilon$.

However, this is not the only problem. Figure 2 shows the many imperfection resonances present due to magnet errors. These errors can in principle be tuned out by using correction dipoles. In fact, this method has been quite successful at the Argonne ZGS and is proposed for the Brookhaven AGS. ${ }^{10}$ To accomplish this the spin itself is used as an indicator to adjust the phase and magnitude of the correction. This is fine so long as the resonances are well separated (or compensated for by tune shifts). However, the large resonance at $60-\nu$ in the AGS is very close to imperfection resonance No. 51. Since they are so close, they probably cannot be crossed separately, and strictly speaking, the theory of isolated resonances does not apply in this case. This remains an unsolved problem.

Finally we should mention an entirely different approach to eliminating resonances by modifying the precession of the spin, the so called Siberian Snake. ${ }^{13}$ In this method one introduces precessions about a horizontal axis which effectively change the spin precession frequency to $1 / 2$ at all energies. In doing so one sacrifices the polarization at most points along circumference in favor of knowing the polarization at one 
point. Although this method is well founded in theory, it has yet to be tested experimentally. A Siberian Snake would be necessary for polarizing a high energy storage ring such as ISABELLE since the resonances would be too numerous to cross effectively; however, that is another story.

\section{REFERENCES}

1. M. Froissart and R. Stora, Nucl. Inst. and Methods, 1, 297-305 (1960):

2. D. Cohen, Rev. Sci. Instr. 33, No. 2, 161-169 (1962).

3. E. D. Courant, BNL Report EDC-45 (1962).

4. V. Ernst, Nucl. Inst, and Methods, 60, 52-60 (1968).

5. T. Khoe, R.L. Kustom, R.L. Martin, E.F. Parker, C.W. Potts, L.G. Ratnor, R.E. Timm, A.D. Krisch, J.B. Roberts, J.R. O'Fallon, Particle Accelerators, 6, No. $4,213-236$ (1975).

6. See University of Michigan preprint HE80-2.

7. E.D. Courant, AIP Conf. Proceedings, No. 42, p. 94. (1977).

8. V. Bargman, L. Michel and V.L. Telegdi, Phys. Rev. Lett. 2, 435 (1959).

9. B.W. Montague, CERN Report, CERN/ISR-TH/80-02 (1980).

10. D.G. Crabb, A.D. Krisch, K.M. Terwilliger, B. Cork, E.D. Courant, A. Feltman, R.D. Ruth, E.F. Parker, L.G. Ratner, IEEE Transactions on Nuclear Science, NS-26, No. 3, 3203-3205.

11. E.D. Courant and H.S. Snyder, Ann. Physics 3, 1-48 (1958).

12. A. Turrin, LNF Report, LNF-78/53(P) (1978).

13. Ya. S. Derbenev and A.M. Kondratenko, Proceedings of the 10 th International Conference on High Energy Accelerators, Protvino, Vol. 2, p. $70(1977)$. 